\title{
Atypical band keratopathy following long-term pilocarpine treatment
}

\author{
D J BRAZIER AND $R$ A HITCHINGS \\ From Moorfields Eye Hospital, High Holborn, London WC1 7AN
}

SUMMARY Two patients with an atypical form of band keratopathy following long-term pilocarpine treatment are described. The keratopathy is thought to have resulted from the presence of the preservative phenylmercuric nitrate in the pilocarpine drops. Symptoms of reduced acuity, visual haloes, and recurrent epithelial erosions were relieved by removal of the opacities.

In 1971 Kennedy et al. ${ }^{1}$ drew attention to a form of band keratopathy occurring in glaucoma patients on miotics. The keratopathy was thought to result from exposure to phenylmercuric nitrate, the preservative agent in the drops used. ${ }^{2}$ More recently Kenyon et al. ${ }^{3}$ wrote that instillation of mercury-containing eye drops, as in glaucoma and dry-eye states, has some circumstantial relationship to the development of band keratopathy in some patients'. This paper records two cases of band keratopathy following long-term use of pilocarpine drops containing phenylmercuric nitrate.

\section{Case reports}

\section{CASE 1}

This patient initially attended in 1955 when aged 31 . She complained of intermittent visual haloes associated with ocular pain. Visual acuities were normal, but the anterior chambers were shallow, with narrow angles on gonioscopy. Intraocular pressures and optic discs were normal, as were the other results of the ophthalmic examination. A diagnosis of intermittent angle closure glaucoma was made and treatment started with pilocarpine $0.5 \%$ drops twice daily to both eyes. The patient remained on this treatment without new symptoms or signs until 1982, a period of 27 years.

In 1982 she reported having blurred vision when looking towards bright lights, and she was found to have a faint opacity in the right cornea, inferonasal to the visual axis, at the level of the superficial stroma. An isolated crystalline deposit was in the inferonasal quadrant of the left cornea. Visual acuities were

Correspondence to Mr D J Brazier, Department of Ophthalmology University College Hospital, Gower Street, London WC1. normal. During the next two years the corneal opacity increased in size and density in the right eye and a similar opacity appeared on the left. Serum calcium was normal.

The patient underwent bilateral laser iridotomy in 1984. During the following months she made a number of unscheduled visits to hospital complaining of haloes but each time had normal or minimally raised intraocular pressures and no corneal oedema. In addition the right acuity was now reduced, varying between $6 / 6$ and $6 / 12$ at different visits. There was quite marked granular yellow-brown discolouration of the cornea, maximal in the subepithelial zone, in a band shaped distribution (Fig. 1). Slit-lamp examina-

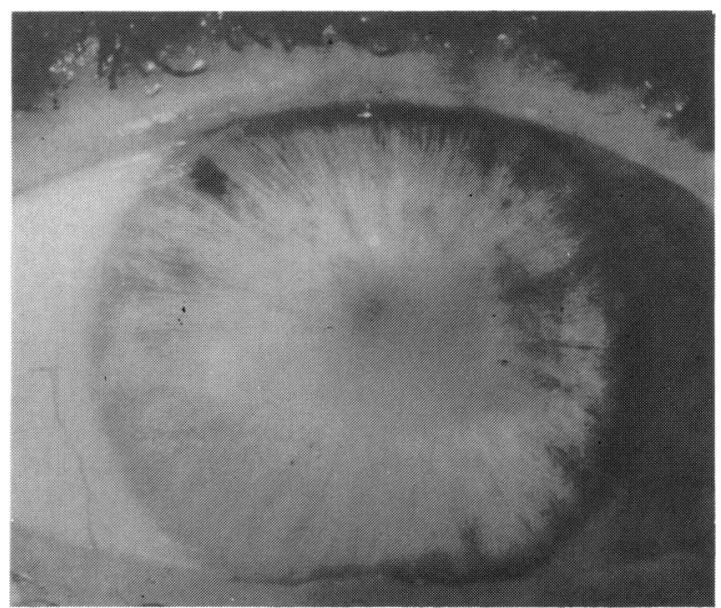

Fig. 1 Case 1, right eye. Band keratopathy obscuring pupil margin and central iris detail. Laser iridotomy visible adjacent to the upper lid margin. 
tion did not identify any associated changes in the anterior lens capsule of either eye.

The band keratopathy was removed under local anaesthesia in May 1985 with $0.37 \%$ edetic acid (EDTA) solution. The corneal epithelium healed without complication, acuity was restored to $6 / 5$, and the visual haloes ceased. Changes in the left eye have not so far warranted surgical treatment.

\section{CASE 2}

This man was found to have primary open-angle glaucoma in 1958 and was treated with topical pilocarpine $0.5 \%$ three times daily and adrenaline $2 \%$ once daily. In 1963 faint opacities were noted in the nasal part of both corneas not extending to the limbus. During the following four years the opacities increased in density and occupied an increasing area of nasal and inferior cornea.

Because of impairment of visual acuity and recurrent episodes of irritation, the band keratopathy was removed from each eye by superficial keratectomy in 1967. The corneal changes recurred and required further intervention over succeeding years to maintain clarity of the visual axis or remove flakes of calcific material causing epithelial erosions. Throughout this period the patient used pilocarpine drops 0.5 or $1 \%$ three times a day in addition to other glaucoma treatments including adrenaline, adrenaline with guanethidine, dipivalyl adrenaline, and timolol. The serum calcium was normal.

Since 1984 argon laser trabeculoplasty and bilateral trabeculectomy have been required to control the intraocular pressure. At the most recent follow-up some pigment stippling of the anterior lens capsule was noted in each eye, though this was not associated with any generalised discolouration of the lens. Corrected visual acuities were right $6 / 12$, left $6 / 9$, with upper and lower arcuate field loss in both eyes.

\section{Discussion}

Band keratopathy is commonly seen in association either with a variety of other eye conditions, including uveitis, absolute glaucoma, and phthisis, or with systemic hypercalcaemia. ${ }^{34}$ These causes are unlikely in the patients reported here; band keratopathy would be unusual in controlled glaucoma with good vision, and both patients were normocalcaemic. Primary (or idiopathic) band keratopathy ${ }^{5}$ is another possibility. As neither patient gave a family history of corneal disease, considered important in diagnosis by Duke-Elder and Leigh, ${ }^{4}$ this must also be considered unlikely.

The reported cases do, however, show features of the atypical form of band keratopathy initially described by Charney ${ }^{6}$ and later by Kennedy et al. ${ }^{1}$ The latter authors reported on 18 well controlled glaucoma patients who developed fine granular opacities in the lower central and nasal parts of the cornea. This band keratopathy was considered atypical because it began centrally and gradually extended towards the corneal periphery rather than starting at the limbus and extending centrally, as is more usual. ${ }^{4}$ All affected patients were using miotics. In patients using miotics in only one eye the band keratopathy was restricted to the treated eye.

A further publication ${ }^{2}$ contained a detailed analysis of the constituents of pilocarpine drops used by a total of 48 patients with the atypical band keratopathy. It transpired that all affected individuals used pilocarpine made by one manufacturer, which differed from other brands available in containing phenylmercuric nitrate as a preservative agent. It was concluded that the keratopathy resulted from the use of pilocarpine drops containing phenylmercuric nitrate, though no precise explanation of the mechanism was possible.

Both our patients were treated with pilocarpine preserved with phenylmercuric nitrate for many years, though this agent was not used in pilocarpine made at Moorfields Eye Hospital after the mid 1970s. Interestingly, both patients continued to develop corneal opacities after exposure to phenylmercuric nitrate ceased. This suggests either that phenylmercuric nitrate continues to predispose to development of band keratopathy after it has been discontinued or that another, as yet unidentified, factor is involved.

The theory that phenylmercuric nitrate has a significant role in development of the band keratopathy is supported by other reports of corneal opacification following exposure to both elemental mercury and phenylmercuric nitrate. Band keratopathy was reported in a barometer maker by Nettleship ${ }^{5}$ and in thermometer makers examined by Atkinson ${ }^{7}$ and Burn. ${ }^{8}$ These occupations involved exposure to mercury vapour. Abrams, in his study of iatrogenic mercurialentis published in $1963,{ }^{\circ}$ reported band keratopathy in five patients treated with miotics containing phenylmercuric nitrate. Two of these patients had unexplained band keratopathy without signs of mercurialentis. Corneal changes in the other three cases could be explained by absolute glaucoma.

Neither patient reported on here was considered to show signs of mercurialentis, the grey-brown or yellow discolouration of the anterior lens capsule which may follow exposure to mercury or phenylmercuric nitrate. ${ }^{7-10}$ The pigment deposits on the anterior lens capsule of case 2 were thought to be compatible with pigment release in the anterior 
segment after bilateral drainage surgery. Grant ${ }^{10}$ noted that the report by Kennedy et al. ${ }^{2}$ associating atypical band keratopathy with phenylmercuric nitrate made little mention of lens changes. In the same way relatively few of the reported patients with mercurialentis following use of drops containing phenylmercuric nitrate had corneal signs. ${ }^{9}$ It therefore seems that the corneal and lens changes appear more often in isolation than together.

Reported symptoms of the atypical band keratopathy ${ }^{126}$ include visual disturbance, photophobia, lacrimation, and irritation, the last three when erosion of the overlying epithelium occurs. Our first patient also suffered visual haloes caused by the granular opacity in the cornea. When the band keratopathy gives rise to symptoms, removal with edetic acid solution, as described by Grant," is effective. The procedure can be repeated in the event of recurrence.

While no longer used in pilocarpine, phenylmercuric nitrate is still present in some drops prescribed for glaucoma and antibiotic preparations. The link should be borne in mind when patients develop band keratopathy during long-term topical treatment.

We thank Mr P Fells for allowing us to report a patient under his care (case 1) and the Departments of Medical Illustration at Moorfields
Eye Hospital and St Thomas's Hospital, which respectively took and prepared the photograph for publication.

\section{References}

1 Kennedy RE, Roca PD, Landers PH. Atypical band keratopathy in glaucomatous patients. Am J Ophthalmol 1971; 72: 917 22.

2 Kennedy RE, Roca PD, Platt DS. Further observations on atypical band keratopathy in glaucoma patients. Trans $\mathrm{Am}$ Ophthalmol Soc 1974; 72: 107-19.

3 Kenyon KR, Fogle JA, Grayson M. Dysgeneses, dystrophies and degenerations of the cornea. In: Duane TD, ed. Clinical ophthalmology. Philadelphia: Harper and Row, 1983: 4: chapter 16: 41-3.

4 Duke-Elder S, Leigh AG. Diseases of the outer eye. In: DukeElder S, ed. System of ophthalmology. London: Kimpton, 1965 $8(2)$ : 893-902.

5 Nettleship E. On a rare form of primary opacity of the cornea. Arch Ophthalmol 1879; 8: 293-319.

6 Charney SM. Idiopathic band keratopathy. Arch Ophthalmol 1966; 75: 505-7.

7 Atkinson WS. A colored reflex from the anterior capsule of the lens which occurs in mercurialism. Am J Ophthalmol 1943; 26: 685-8.

8 Burn RA. Mercurialentis. Proc $R$ Soc Med (Ophthalmological Section) 1962-3; 55-56: 322-6.

9 Abrams JD. Iatrogenic mercurialentis. Trans Ophthalmol Soc UK 1963; 83: 263-9.

10 Grant WM. Discussion. Further observations on atypical band keratopathy. Trans Am Ophthalmol Soc 1974; 72: 119-21.

11 Grant WM. New treatment for calcific corneal opacities. Arch Ophthalmol 1952; 48: 681-5.

Accepted for publication 12 May 1988. 